(Aus dem pharmakologischen Institut der Reichsuniversität Utrecht.)

\title{
Über die Wirkung von Äther auf Säugetierherz und Kreislauf.
}

Von

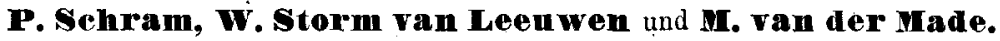

(Mit 6 Textfiguren.)

Nachdem der eine von uns (S. v. L.) ${ }^{1}$ ) in einer früheren Untersuchungsreihe den Einfluss des Äthers auf die Reflextätigkeit des Rückenmarkes studiert und dabei den Äthergehalt des Blutes und des Rückenmarkes in verschiedenen Stadien der Narkose bestimmt hatte, ergab sich die Frage, welchen Einfluss die hierbei in Betracht kommenden Ätherkonzentrationen auf den Zirkulationsapparat ausüben. Ein derartiger Vergleich zwischen der Wirkung auf Rückenmarksfunktionen und Herz war für das Chloroform möglich gewesen, weil Sherrington and Sowton ${ }^{2}$ ) nach Versuchen an isolierten, mit unverdünntem Blute durchströmten Katzenherzen die genauen Chloroformkonzentrationen angeģeben hatten, welche das Herz zu schädigen resp. zum Stillstand zu bringen imstande waren. Für Äther aber lagen analoge Untersuchungen nicht vor. Zwar gab es eine Anzahl Arbeiten über die Wirkung des Äthers auf das Herz, aber in keinem dieser Versuche war mit unverdünntem Blute gearbeitet worden, so dass die gefundenen Zahlen für unsere Zwecke keinen Wert hatten. Eine kurze Übersicht der Literatur möge dieses veranschaulichen.

1) Quantitative pharmakologische Untersuchungen über die Reflexfunktionen des Rückenmarkes an Warmblütern. III. Mitteilung: Wirkung von Äther. Pflüg er's Arch. Bd. 165 S. 84. 1916.

2) C. S. Sherrington and S. C. M. Sowton, On the dosage of the isolated mammalian heart by chloroform. The British Med. Journ. vol. 2 p. 162.1904. 


\section{Literaturübersicht.}

Die ältesten hier zu berücksichtigenden Versuche stammen von D i e $b$ all $a^{1}$ ). Er durchströmte isolierte Froschherzen mit NaCl-Lösung und fügte dieser Lösung steigende Mengen Äther zu. Ein Äthergehalt von $0,235 \%$ war in seinen Versuchen ohne Einfluss auf das Herz; bei. $0,47 \%$ war eine Wirkung wahrnehmbar, die Kontraktionen wurden kleiner; aber es war ein Gehalt von $2,84 \%$ nötig, um innerhalb 21/2 Minuten das Herz zum Stillstand zu bringen.

Die ersten Versuche am Warmblüterherzen sind von Bock ${ }^{2}$ ). Er arbeitete am isolierten Herz-Lungenkreislauf des Kaninchens. Das Narkotikum wurde den Tieren mit der Einatmungsluft zugeführt. Er fand: "Trotz der bedeutenden eingeatmeten Äthermengen und trotz ziemlich lange fortgesetzter Inhalation erzeugte der äther in den angestellten Versuchen kein oder geringes Sinken des Blutdrucks, und auch im letzten Falle stieg er nach dem Ausstellen der Inhalation sehr schnell wieder bis zu seiner ursprünglichen Höhe an."

Leider vermeldet Bock nur die Dauer der Ätherinhalation, nicht aber den Äthergehalt der Einatmungsluft oder des Blutes.

$\mathrm{Pick}^{3}$ ) veröffentlichte im nächsten Tahr eine Arbeit über die Gefässwirkung verschiedener Gifte. Er bestimmte den Einfluss auf Blutdruck und Geschwindigkeit des Blutstroms. Chloroform verursachte Gefässerweiterung im Abdomen und Gehirn und Blutdrucksenkung, Äther „bei zur Narkose genügenden Mengen wohl keine so hochgradige Blutdrucksenkung, aber analoge Veränderungen der Ausflussmengen aus den Venen". Angaben über die Konzentrationen des Äthers im Blute fehlen, nur gibt er an, dass zum Beispiel in einem Versuche an einem Hund, wo der Blutdruck im Anfang des Versuches $146 \mathrm{~mm} \mathrm{Hg}$ betrug, derselbe nach Einatmung von Äther bis auf 112 sank und in einem Stadium, wo die "Reflexe geschwunden waren", zwischen 95, 88 und 109 schwankte.

Elfstrand $\mathbf{d}^{4}$ ) studierte die Wirkung verschiedener Narkotika auf den Blutdruck bei Kaninchen. Er fand, dass Äther auf den Blutdruck dieser Tiere weniger Einfluss ausübt als andere Narkotika, zum Beispiel Chloroform oder Pentan. Er führt aber keine Beweise dafür an, dass beim Narkotisieren mit den verschiedenen Giften die Narkose immer gleich tief war.

1) G. Dieballa, Über die quantitative Wirksamkeit verschiedener Stoffe der Alkohol- und Chloroformgruppe auf das Froschherz. Arch. f. exper. Pathol. u. Pharmak. Bd. 34 S. 147. 1894.

2) J. Bock, Untersuchungen über die Wirkung verschiedener Gifte auf das

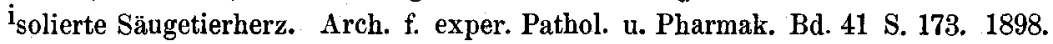

3) F. Pick, Über Beeinflussung der ausströmenden Blutmenge durch die Gefässweite ändernde Mittel. Arch. f. exper. Pathol. u. Pharmak. Bd. 42 S. 399.1899.

4) M. Elfstrand, Beobachtungen über die Wirkung einiger aliphatischer Kohlenwasserstoffe, verglichen mit derjenigen des Äthers, und über das Verhalten der Vaguserregbarkeit während der Narkose. Arch. f. exper. Pathol. u. Pharmak. Bd. 43 S. 435.1900. 
Knol1') hatte schon vor Jahren bei Versuchen an Kaninchen, die er durch eine Trachealfistel Äther und Chloroform einatmen liess, festgestellt, dass „die Erscheinungen an der Blutdruckkurve bei der Inhalation von Äther weit weniger ausgeprägt sind als bei der Chloroforminhalation". Für Tiere mit durchschnittenen Vagi galt "dies in noch weit höherem Maass".

Auch hier wird nicht der exalste Beweis geliefert, dass die Chloroform- und Äthernarkosen gleich tief waren. Nach seinen Versuchen ist es aber sehr wahrscheinlich, dass erstens Äther einen viel geringeren Einfluss auf den Blutkreislauf hat als Chloroform, und zweitens, dass der Einfluss des Äthers sich bei Anwendung mässiger Dosen auf die Vasomotoren beschränkt, während Chloroform in dieser Dosierung schon das Herz schädigt. Einen Beweis für diese Auffassung brachte Mac William²). Er registrierte bei Katzen gleichzeitig den Blutdruck und die Kontraktionen von Vorhof und Kammer und fuhrte dann seinen Versuchstieren durch die künstliche Atmung Chloroform und Äther zu. Er fand, dass bei Dosen, welche ausreichten, um den Kornealreflex verschwinden zu lassen, Äther eine Blutdrucksenkung hervorrief, aber noch keinen Einfluss auf das Herz ausübte, während Chloroform bei dieser Narkosetiefe nicht nur eine Blutdrucksenkung verursachte, sondern auch das Herz schädigte.

Bei einer Untersuchung über die Todesursache bei Chloroform-

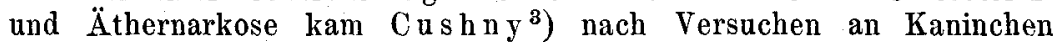
und einigen Hunden, denen er die Narkotika durch die künstliche Atmung zuführte, zu dem Resultat, dass beide Narkotika im Prinzip dieselbe Wirkung haben. "Bei beiden folgt Tod durch Atmungs- oder fast gleichzeitiger Herz- und Atmungslähmung."

In den bis jetzt besprochenen Untersuchungen über die Wirkung des Äthers auf Herz und Kreislauf der Warmblüter fehlen Angaben über die in den Versuchen zur Verwendung kommenden Konzentrationen des Narkotikums.

Tunnicliffe and Rosenheim und 0 . Loeb haben aber in ihren Versuchen an isolierten Säugetierherzen genaue Bestimmungen des Chloroform- oder Äthergehaltes der Durchströmungsflüssigkeit gemacht.

Tunnicliffe and Rosenheim ${ }^{4}$ ) durchbluteten isolierte Säuge-

1) Ph. Knoll, Über die Wirkung von Chloroform und Äther auf Atmung und Blutkreislauf. II. Mitteilung. Sitzungsber. d. Wiener Akad. d. Wissensch. Bd. 78 Abt. 3 S. 223.1878.

2) J. A. MacWilliam, Report on an experimental investigation of the action of Chloroform and ether. British Med. Journ. vol. 2 p. 831. 1890.

3) A. Cushny, Über Chloroform- und Äthernarkose. Zeitschr. f. Biol. Bd. 28 S. 365.1891.

4) F. W. Tunnicliffe and O. Rosenheim, On the action of chloroform, ether, alcohol and acetone upon excised mammalian beart. Journ. of physiol. vol. 29. 1903. Proc. physiol, Soc. p. 15. 
tierherzen mit L ocke's Lösung. Zufügung von $0,01{ }^{\circ} \%$ Chloroform hatte einen merklichen Einfluss, $0,1 \%$ Chloroform brachte das Herz zum Stillstand. Äther wurde nur in Dosen bis zu $0,02 \%$ angewandt. "The effect of weak and strong solutions alike was to produce at first slight irregularity and slowing, but the effect was quite transient.

Schliesslich hat Loe ${ }^{1}$ ) die Wirkung des Äthers auf das isolierte Katzenherz untersucht. Als Durchströmungsflüssigkeit wurde ein BlutKochsalzgemisch benutzt. Loeb fand als minimal schädigende Dosis $0,133 \%$ und als minimal letale Dosis $1,7 \%$. Diese Zahlen sind wesentlich geringer als diejenigen Dieballa's. Dies mag wohl zum Teil seine Erklärung in dem Umstand finden, dass Dieballa am Froschherz, L o eb aber am Warmblüterherz gearbeitet hat, und dabei wird ersterer seine Versuche bei weit niedrigerer Temperatur angestellt haben als Loeb.

Wie aus dieser kurzen Zusammenstellung der Literatur ersichtlich ist, liegen keine Angaben vor über die Äthermengen, welche dem unverdünnten Blute zugefügt werden müssen, um bestimmte Änderungen in der Herztätigkeit hervorzurufen.

Zweck der vorliegenden Arbeit ist, diese Lücke auszufüllen. Auf vier verschiedene Weisen haben wir genaue Ätherzahlen in unverdünntem Blute zu ermitteln versucht:

1. durch Versuche am isolierten Herz-Lungenkreislauf nach Starling;

2. durch Blutdruckversuche am intakten Tiere mit intakten Vagi;

3. durch Blutdruckversuche am intakten Tiere mit durchschnittenen Vagi oder nach Atropineinspritzung;

4. durch Versuche am isolierten Herzen.

\section{Einfluss des Athers auf das Herz bei Versuchen mit isoliertem Herz-Lungenkreislauf nach Starling. Von}

\section{P. W. Schram, W. Storm van Leeuwen und M. van der Made.}

Seit 1912 haben Starling ${ }^{2}$ ) und seine Mitarbeiter eine Reihe von Untersuchungen über die Mechanik des Säugetierherzens veröffentlicht, wobei ein sehr bequemes und zweckmässiges Verfahren

1) O. Loeb, Die Wirkung des Alkohols auf das Warmblüterherz. Arch. f. exper. Pathol. u. Pharmak. Bd. 52 S. 459. 1905.

2) Knowlton and E. H. Starling, The influence of variation in temp. and blood presslire on the performance of the isolated mammalian heart. Journ. of physiol. vol. 44 p. 205.1912. 
benützt wurde, welches sich auch in unserem Institut sehr gut bewährt hat. Fur eine genaue Beschreibung der Methode (isoliertes Herz-Lungenpräparat zum künstlichen Kreislauf) sei auf die ursprüngliche Literatur verwiesen. In der deutschen Literatur ist das Verfahren von $S_{\operatorname{traub}}{ }^{1}$ ) und von Socin ${ }^{2}$ ) beschrieben worden.

Unsere Versuchsanordnung wich nur in zwei Punkten von der Starling'schen Methode ab. Starling arbeitete mit nur teilweise defibriniertem Blut and setzte Hirudin zu, während wir in jedem Versuch das sorgfältig defibrinierte Blut von drei oder vier Katzen verwendeten ohne Zusatz von Hirudin. Eine zweite Abweichung von der Starling-Methode, welche schon von S oc in beschrieben wurde, bestand darin, dass das Zeitvolumen mit dem von $\mathrm{Cond} \mathrm{on}^{3}$ ) beschriebenen Volummesser gemessen wurde.

Registriert wurden in unseren Versuchen:

a) Das Volumen des Herzens. Hierzu war das Herz in einem Glasballon eingeschlossen, über den Ballon war das Perikard gestü]pt. Der Raum zwischen Herz und Perikard stand durch einen weiten Gummischlauch in Verbindung mit einem Pistonrekorder, dessen Ausschläge registriert wurden.

b) Der Druck im künstlichen arteriellen System wurde mit einem gewöhnlichen Quecksilbermanometer registriert.

c) Das Zeitrolumen. Bei Benützung des Condon'schen Volummessers wird jedesmal, wenn $10 \mathrm{ccm}$ Blut das arterielle System durchströmt haben, ein elektrischer Kontakt geschlossen und auf dem Kymographion markiert.

Ein typischer Versuch verlief nun folgendermaassen. Vor dem Anfang der eigentlichen Operation wurden drei oder vier Katzen in Äthernarkose entblutet. Dieses Blut wurde sorgfältig defibriniert and diente zur Füllung des Apparates. Es wurde dann das eigentliche Versuchstier narkotisiert und die Operation nach $\mathrm{S}$ t a r l in g vorgenommen. Meistens arbeitete das Herz, nachdem es am Ende der Operation mit dem Apparat in Verbindung gebracht worden war, sofort tadellos weiter. $A b$ und zu aber trat offenbar infolge der Durchströmung mit dem defibrinierten Blute Bronchialmuskelkrampf auf, so dass fast keine Luft mehr in die Lunge gepresst werden konnte und die Sauerstoffversorgung des Herzens sehr litt. Einige Tropfen Adrenalin, welche dann dem Blute zugesetzt wurden, beseitigten diesen Bronchialmuskelkrampf fast momentan. ${ }^{4}$ )

1) M. Straub, Dynamik des Säugetierherzens. Deutsch. Arch. f. klin. Med. Bd. 115 S. 531.1914.

2) Ch. Socin, Experimentelle Untersuchungen über akute Herzschwäche. Pflüger's Arch. Bd. 160 S. 132.1914.

3) N. C. Condon, A magnet-tipper for recording outflow. Journ. of physiol. vol. 46. Proc. physiol. Soc. Jun. $28^{\text {th }}$. 1913.

4) Siehe Socin, a. a. O. 
Nachdem eine genügend lange Normalperiode registriert worden war, wurde den Lungen mittels der Einatmungsluft Äther zugeführt, erst in kleineren, später in grösseren Konzentrationen. Der Einfluss des Äthers war bei den kleineren Konzentrationen (wie sie im Laboratorium gewöhnlich zu Narkosezwecken benützt werden) sehr gering, es trat meistens eine geringe Dilatation des Herzens auf, der Blutdruck blieb normal, das Zeifvolumen änderte sich wenig oder gar nicht. Wurden dagegen grössere Mengen Äther angewandt, so dilatierte das Herz immer mehr, das Zeitvolumen nahm ab, der Blutdruck sank, und bei noch böheren Konzentrationen traten Pulsunregelmässigkeiten auf. Selbst wenn die ganze Einatmungsluft, ehe sie die Lungen erreichte, durch Äther gestrichen war, gelang es noch nicht, so hohe Ätherwerte im Blute zu erhalten, dass das Herz dadurch zum Stillstand gebracht wurde. Manchmal wurde deshalb in das venöse Reservoír des Apparates reiner Äther gegessen. Durch diese sehr grosse Äthermenge wurde das Blut dann meistens hämolytisch, aber trotzdem reichte die Dosis manchmal nicht aus (wiewobl die Herztätigkeit natürlich sehr geschädigt wurde), um das Herz ganz zum Stillstand zu bringen.

Es sei hierbei bemerkt, dass Langendorff und sein Schüler Brandenburg gezeigt haben, dass im Gegensatz zu Kaninchenblut lackfarbenes Katzenblut für das isolierte Katzenherz unschädlich ist. „Cytolytisches Blut von solcher Beschaffenheit vermag mehrere Stunden lang ein ausgeschnittenes Katzenherz in guter und regelmässiger Tätigkeit zu erhalten." "1) Die bekannte schädliche Wirkung des hämolytischen Blutes verschiedener Tierarten beruht auf dem Kalireichtum der Blutkörperchen. Die Blutkörperchen des Hundes und der Katze enthalten nur wenig Kali, diejenigen des Menschen, Kaninchens und des Schweines aber sehr viel. Demgemäss ist das cytolytische Hunde- und Katzenblut nicht giftig. Dagegen sind die anderen Blutarten, wenn sie lackfarbig geworden sind, für das isolierte Säugetierherz schädlich.

In bestimmten Phasen der Ätherwirkung wurde dem Apparate Blut entnommen und der Äthergehalt desselben chemisch bestimmt. Es kam dabei die von Nicloux ${ }^{2}$ ) ausgearbeitete Methode zur Verwendung. Diese Methode hat sich - wie schon in einer früheren

1) O. Langendorff, Über die angebliche Unfähigkeit des lackfarbenen Blutes, den Herzmuskel zu ernähren. Pflüger's Arch. Bd. 93 S. 286. 1903.

2) M. Nicloux, Les anesthésiques généraux au point de vue chimicophysiologique. Paris 1908. 
Über die Wirkung von Äther auf Säugetierheř und Kreislauf.

Arbeit von einem von uns mitgeteilt wurde ${ }^{1}$ ) - im Institut sehr gut bewäbrt.

Es wurde in vier Stadien der Ätherwirkung den Tieren Blut entnommen:

a) in drei Fällen vor dem Zusatz von Äther (Koptrollversuch);

b) im Stadium der beginnenden Schädigung;

c) im Stadium der stark ausgesprochenen Schädigung;

d) im Stadium, wo Herzstillstand aufgetreten war oder das Herz zwar noch schlug, aber so stark geschädigt worden war, dass es $\mathrm{zu}$ wesentlicher Arbeitsleistung nicht mehr imstande war. Das Resultat der Ätherbestimmungen ist aus Tabelle I ersichtlich.

$$
\text { Tabelle I. }
$$

Versuche am isolierten Herz-Lungenkreislauf nach Starling.

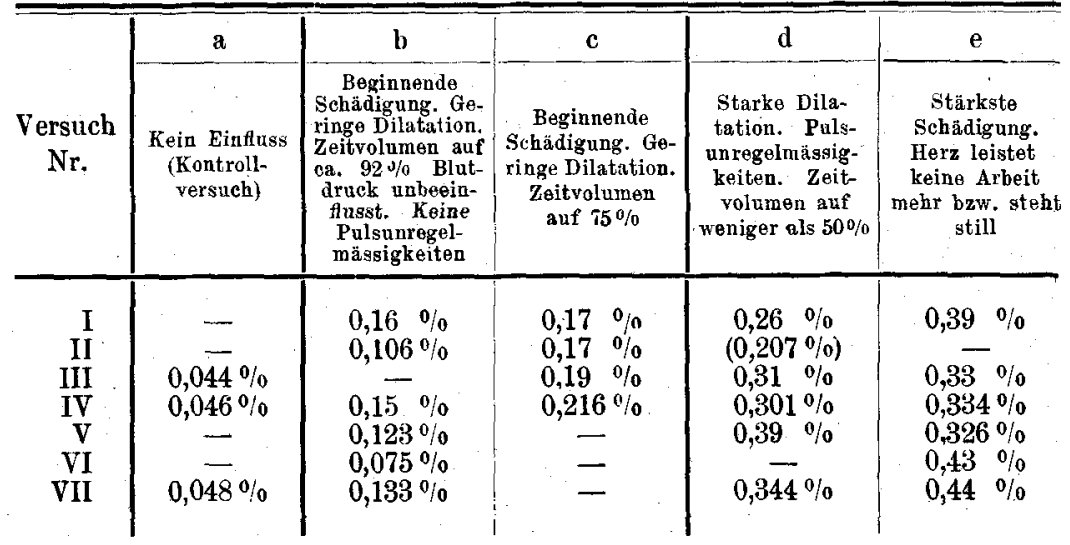

$\mathrm{Zu}$ dieser Tabelle ist folgendes zu bemerken:

a) Kontrollbestimmungen über den Äthergehalt des Blutes vor dem Anfang des eigentlichen Versuches waren notwendig, weil das in den Versuchen zur Verwendung kommende Blut immer Tieren entstammte, welche in Äthernarkose entblutet worden waren. In drei Fällen wurde dieser Wert bestimmt, wobei $0,044 \%, 0,046 \%$ und $0,048 \%$ gefunden wurden, im Mittel also $0,046 \%$. Dieser Wert ist im Vergleich mit den später in Betracht kommenden Mengen

1) W. Storm van Lee uwen, Quantitative pharmakologische Untersuchungen über die Reflexfunktionen des Rückenmarkes an Warmblütern. III. Mitteilung. Pflüg er's Arch. Bd. 165 S. 84.1916.

Pflüger's Arehiy für Physiologie. Bd. 165. 
130 P. Schram, W. Storm van Leeuwen und M. van der Made:

so niedrig, dass das Resultat der weiteren Versuche durch diese kleinen Äthermengen im Blute in keinerlei Weise beeinträchtigt werden kann.

b) Eine beginnende Schädigung des Herzens wurde angenommen, wenn das Herz anfing zu dilatieren, der Blutdruck aber noch normal

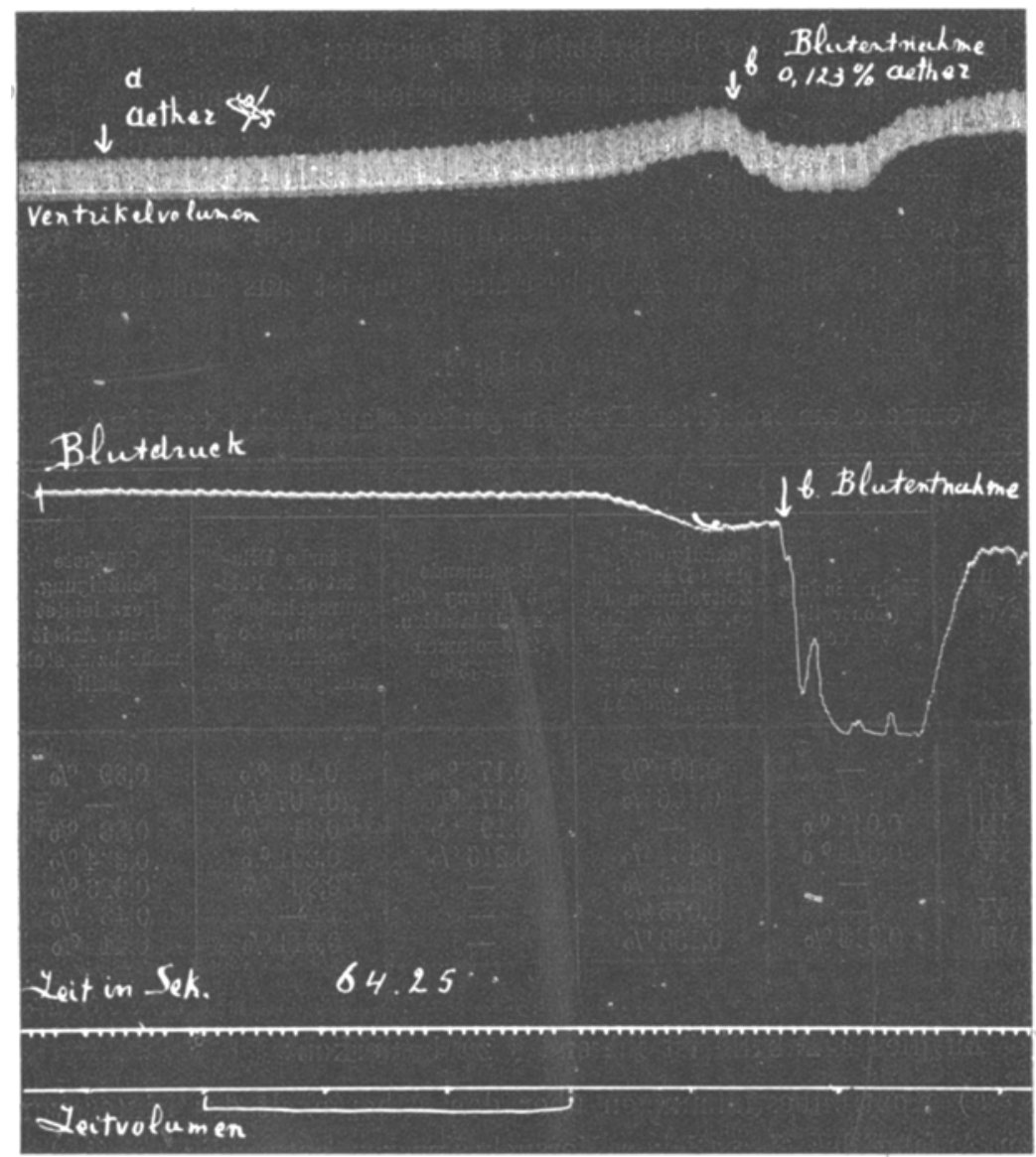

Fig. 1. Isolierter Herz-Lungenkreislauf nach Starling. Versuch V. Beginnende Schädigung des Herzens durch $0,123 \%$ Äther. Bei $a$ Einschaltung einer mässigen Ätherkonzentration. Geringe Dilatation des Herzens. Zeitvolumen und Blutdruck wenig beeinflusst. Bei $b$ Blutentnahme. Die Anderungen im Ventrikelvolumen und Blutdruck nach $b$ sind durch die Blutentnahme vorgetäuscht. (Auf $\$ / 3$ verklejnert.)

war, keine Pulsunregelmässigkeiten aufgetreten waren und das Zeitvolumen nicht niedriger als bis auf ca. $92 \%$ des ursprünglichen Wertes heruntergegangen war. In diesem Stadium war der Äthergehalt als Minimum $\mathbf{0 , 0 7 5} \%$ als Maximum 0,16\% (s. als Beispiel 
Fig. 1). Zwischen diesem Stadium der beginnenden Schädigung und dem nächsten Stadium der schweren Schädigung liegen einige Werte (Spalte c), bei welchen das Herz ebenfalls ein wenig dilatiert, der Blutdruck ein wenig herabgesunken, auch noch keine Pulsunregelmässigkeiten aufgetreten waren, aber das Zeitvolumen schon bis auf

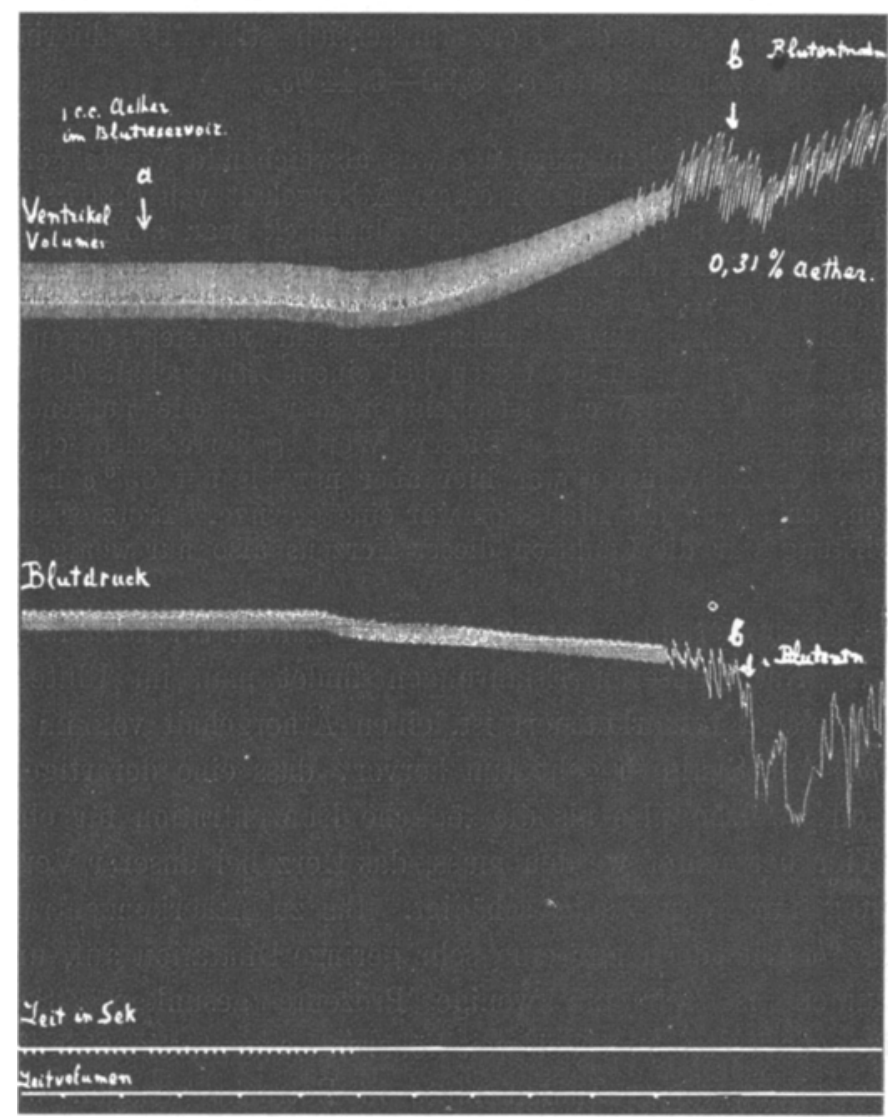

Fig. 2. Isolierter Herz-Lungenkreislauf nach Starling. Versuch III. Starke Schädigung des Herzens durch $0,31 \%$ Äther. Bei "a Zufuhr. einer grossen Menge Äther. Starke Dilatation des Herzens. Auftreten von Pulsunregelmässigkeiten. Senkung des Blutdrucks. - Das Zeitvolumen nimmt ab. Die Schwankungen in der Ventrikel- und Blutdruckkurve nach $b$ sind teilweise durch die Blutentnahme verursacht. (Auf $1 / 2$ verkleinert.)

$75-90 \%$ des Anfangswertes heruntergegangen war. Die Ätherwerte betrugen $0,17-0,216 \%$.

d) Im Stadium schwerer Schädigungen war das Herz stark dilatiert, der Blutdruck gesunken, das Zeitvolumen auf weniger als 
die Hälfte des ursprünglichen Wertes zurückgegangen und Pulsunregelmässigkeiten aufgetreten. Die Ätherwerte betrugen $\mathbf{0 , 2 6}$ bis 0,39\% (s. als Beispiel Fig. 2).

e) Im letzten Stadium, schwerster Sehädigung, schlug das Herz meistens zwar noch, war aber nicht mehr imstande, die Zirkulation zu unterhalten. Die äussere Arbeit. war also auf 0 heruntergegangen. In einem Falle stand das Herz tatsächlich still. Die hierbei gefundenen Ätherzahlen betrugen $0,33-0,44 \%$.

Bei zwei Versuchen wurden etwas abweichende Werte gefunden. In Versuch II waren schon bei einem Äthergehalt von $0,207 \%$ Pulsunregelmässigkeiten aufgetreten, der Blutdruck war auf ca. $20 \mathrm{~mm}$ herabgesunkell, und das Zeitvolumen betrug nur noch $18 \%$ des ursprünglichen Wertes. Dieses Herz war offenbar sehr empfindlich gegen Äther. Ein Beispiel eines Herzens, das sehr resistent gegen Äther war, gab Versuch V. Hier traten bei einem Äthergehalt des Blutes von $0,267 \%$ (dieser Wert ist nicht in der Tabelle aufgenommen) Pulsunregelmässigkeiten auf. Dieser Wert gehörte also eigentlich unter c. Das Zeitvolumen war hier aber nur bis auf $85 \%$ heruntergegangen, und auch die Dilatation war eine geringe. Trotz erheblicher Ätherwirkung war die Funktion dieses Herzens also nur wenig gestört.

Bezüglich der hier gefundenen Ätherzahlen sei nocb folgendes bemerkt. Nach unseren Erfahrungen findet man im Blute eines Tieres, welches totnarkotisiert ist, einen Äthergehalt von im Mittel $0,164 \%$. Aus Spalte b geht nun hervor, dass eine derartige Konzentration, welche also als die tödliche Konzentration für ein normales Tier betrachtet werden muss, das Herz bei unserer Versuchsanordnung nur sehr wenig schädigt. Bis zu Ätherkonzentrationen von $0,16 \%$ tritt doch nur eine sehr geringe Dilatation auf, und das Zeitvolumen ist nur um wenige Prozente gesunken. Schwere Schädigungen treten erst bei weit höheren Konzentrationen auf. Bei der Beurteilung dieser Zahlen muss in Betracht gezogen werden, dass das Herz sich bei unserer Versuchsanordnung unter sehr günstigen Bedingungen befindet, weil es bei konstanter Blutzufuhr immer gegen einen konstanten Druck arbeitet. Wir haben den Widerstand im künstlichen Kreislauf immer auf ca. $80 \mathrm{~mm} \mathrm{Hg}$ eingestellt, weil sich aus. den Versuchen von Starling ergeben bat, dass bei diesem Druck das Herz unter den erreichbar günstigsten Bedingungen arbeitet. Starling wies auch nach (und wir haben uns in unseren Versuchen davon überzeugen können), dass niedrigere Drucke vom Herzen schlecht vertragen werden. Dies hat offenbar 
seinen Grund in dem Umstand, dass bei niedrigem arteriellen Druck das Koronarsystem nicht genügend durehblutet wird und infolgedessen die Sauerstoffversorgung des Herzens leidet, wodurch dasselbe sebr geschädigt wird. Wie schon bemerkt wurde, haben wir in unseren Versuchen den arteriellen Druck auf ca. $80 \mathrm{~mm}$ Hg gehalten. Beim Narkotisieren eines intakten Tieres aber sinkt der Blutdruck allmählich, anfangs infolge der Lähmung des vasomotorischen Zentrums, später auch durch direkte Wirkung des Narkotikums auf das Herz. Sowie aber nun der Blutdruck unter einen bestimmten Wert gesunken ist - und dieser Wert wird beim intakten Tier auch wohl in der Nähe von $80 \mathrm{~mm} \mathrm{Hg}$ liegen -, addiert sich zu der Wirkung des Äthers auf das Herz noch die sehädliche Wirkung eines ungenügenden Koronarkreislaufes.

Es liess sich also erwarten, dass beim intakten Tier - auch bei künstlicher Ventilation der Lungen - schon geringere Äthermengen genügen würden, um das Herz zum Stillstand zu bringeu, als bei Versuchen am Starling-Apparat. Wie sich im nächsten Abschnitt herausstellen wird, war dies in der Tat der Fall.

Bei Versuchen am Starling-Apparat mit unverdüntem Blute wird also durch einen Äthergehalt des Blutes von $0,046 \%$ das Herz nicht beeinflusst, beginnende Schädigung tritt auf bei $0,16 \%$, sehr starke Schädigung bei $0,26 \%$. Die tödliche Konzentration wurde meistens nicht erreicht, sie muss etwa bei $0,4 \%$ liegen.

Zur Beantwortung der Frage, welche Ätherkonzentration beim intakten Tier mit künstlicher $A$ tmung als die tödliche für das Herz zu betrachten sei, wurden die jetzt zu beschreibenden Versuche angestellt.

\section{Versuche am intakten Tiere bei künstlicher Atmung mit und ohne Vagotomie.}

\section{Von}

W. Storm van Leeuwen und M. van der Made.

Die Versuchsanordnung in dieser Serie war die gewöhnliche. Die Tiere (Katzen) wurden narkotisiert, dann wurde in die Trachea eine Trachealkanüle eingebunden und die künstliche Atmung eingeschaltet. In beide Karotiden wurde eine Glaskanüle eingebunden; 
die eine Kanüle diente zur Registrierung des Blutdruckes, die zweite zur Blutentnahme. In den ersten Versuchen blieben die Vagi intakt, in den späteren wurden sie durchschnitten. Nachdem sich herausgestellt hatte, dass zwischen den Versuchen mit und ohne Vagotomie ein deutlicher Unterschied wahrnehmbar war, wurden noch einige Versuche an Tieren mit intakten Vagi, denen Atropin eingespritzt wurde, angeschlossen.

\section{Versuche an Katzen mit intakten Vagi.}

In diesen Versuchen wurde, ehe mit der Registrierung des Blutdruckes anoefangen wurde, die Äthernarkose abgestellt, und wir liessen die Tiere teilweise aufwachen. Dann wurde mit der Registrierung des Blutdruckes begonnen, und nachdem der meistens sehr hohe Blutdruck einigermaassen konstant geworden war, wurde dem Tiere gleich eine ziemliche Konzentration Äther mit der Einatmungsluft zugeführt, welche um ein weniges diejenige uberstieg, die im Institut zu gewöhnlichen Narkosezwecken meistens benützt wird. Meistens erfolgte unmittelbar nach Einschalten der Narkose ein allmähliches Absinken des Blutdruckes, welcher sich dann gelegentlich auf ein neues Niveau einstellte. War dies der Fall, so wurde die Ätherzufuhr vermehrt; dies hatte dann ein neues Herabsinken zur Folge, und schliesslich stellte sich der Blutdruck meistens auf ein Niveau von $40-60 \mathrm{~mm} \mathrm{Hg}$ ein. Wenn nun die Ätherzufuhr so reguliert wurde, dass dem Tiere ausschliesslich solche Luft zugeführt wurde, welche durch Äther gestrichen war, so sank bisweilen der Blutdruck noch mehr, und das Herz wurde zum Stillstand gebracht. Oft aber trat selbst bei dieser Ähermenge kein Herzstillstand ein und wurde noch ein Druck von $40-60 \mathrm{~mm} \mathrm{Hg}$ aufrechterhalten. Es wurde dann die Ätherflasche in ein Gefäss mit Wasser von ca. $30^{\circ} \mathrm{C}$. gestellt. Dies hatte ein sofortiges und schnelles Herabsinken des Blutdruckes zur Folge, und im Augenblicke, wo fast gar keine Schwankungen mehr im. Manometer sichtbar waren, also ganz kurz bevor das Herz stillstand, wurde Blut entnommen.

Bezüglich dieser Blutentnahme sei folgendes bemerkt. Die Entnahme geschah aus einer der Karotiden des Tieres. Stand das Herz dabei noch nicht ganz still, so gelang es gelegentlich, die zur Ätherbestimmuug notwendigen $10 \mathrm{ccm}$ Blut zu gewinnen. Geschah die Entrahme dann sehr schnell, so ergab die chemische Bestimmung des Äthergehaltes nahezu den exakten Wert im Moment des Todes. 
Es ist ein solcher Wert in der Tabelle mit $( \pm)$ bezeichnet. Stand aber das Herz bei der Blutentnahme schon still, so musste Thoraxmassage gemacht werden, und die Entnahme dauerte etwas länger. Wurde die künstliche Atmung während dieser Zeit nicht abgestellt, so wurde, weil die Einatmungsluft einen sehr hohen Äthergehalt hatte, im Blute ein zu hoher Wert gefunden. Es sind diese Zahlen in der Tahelle mit $(+)$ bezeichnet. Wurde schliesslich während der Blutentnahme die künstliche Ventilation abgestellt, oder gelang es nicht, genügende Mengen Blutes aus der Arterie zu bekommen, so dass auch venöses Blut zur. Verwendung kam, so wurde selbstverständlich ein etwas zu niedriger Wert gefunden, denn ein Teil des in dieser Weise gewonnenen Blutes hatte im Körper schon Äther abgegeben und hatte nicht während der Passage durch die Lungen sich von neuem mit Äther beladen können. Derartige Werte sind mit (-) bezeichnet.

Es wurden in dieser Weise acht Versuche angestellt. Eine Übersicht der gefundenen Ätherwerte gibt Tabelle 2.

Tabelle 2 .

Versuche an intakten Katzen mit intakten Vagi. Äthergehalt des Blutes.

\begin{tabular}{|c|c|c|c|c|c|c|}
\hline $\begin{array}{l}\text { Ver- } \\
\text { such } \\
\mathrm{Nr} \text {. }\end{array}$ & $\begin{array}{c}\text { Blutdruck } \\
\text { unbeein- } \\
\text { flusst }^{1} \text { ) } \\
0 \%\end{array}$ & $\begin{array}{c}\text { Blutdruck } \\
\text { bis } \\
\text { auf drei } \\
\text { Viertel }{ }^{\mathbf{1}} \text { ) } \\
\% / 0\end{array}$ & $\begin{array}{c}\text { Blutdruck } \\
\text { bis } \\
\text { auf die } \\
\text { Hälfte }^{1} \text { \} } \\
\% \%\end{array}$ & $\begin{array}{c}\text { Ischiadicus- } \\
\text { Reizung obne } \\
\text { Einfluss auf } \\
\text { Blutdruck } \\
\%\end{array}$ & $\begin{array}{c}\text { Max. farad. } \\
\text { Reizung ohne } \\
\text { Einfluss auf } \\
\text { Blutdruck } \\
\mathbf{0} \%\end{array}$ & $\begin{array}{c}\text { Herz- } \\
\text { stillstand } \\
\%_{0}\end{array}$ \\
\hline I & - & - & $(0,180)$ & - & - & - \\
\hline II & - & - & $\frac{118}{66} 0,101$ & - & - & $0,202(-)$ \\
\hline III & - & $\frac{206}{155} 0,136$ & $\frac{206}{100} 0,172$ & - & - & $0,190(-)$ \\
\hline VII & $\frac{152}{152} 0,11$ & 一 & - & 0,11 & - & $0,207(-)$ \\
\hline VIII & - & - & $\frac{102}{60} 0,212$ & - & 0,212 & $0,270(+)$ \\
\hline XI & - & $\frac{170}{126} 0,138$ & - & 0,138 & - & $0,237(-)$ \\
\hline XII & - & $\frac{98}{74} 0,206$ & - & - & 0,206 & $0,323(+)$ \\
\hline XIII & - & - & $\frac{130}{64} 0,188$ & - & 0,183 & $0,250( \pm)$ \\
\hline
\end{tabular}

1) Die Bruchzahlen beziehen sich auf den Blutdruck. Der Zähler gibt den Anfangsblutdruck, der Nénner den Blutdruck im Augenblick der Blutentnahme. 
136 P. Schram, W. Storm van Leeuwen und M, van der Made:

In den Versuchen dieser Tabelle ist viermal Blut entnommen worden im Stadium, wo der Blutdruck auf zirka die Hälfte der Anfangshöhe heruntergegangen war. Die Werte schwanken von $0,101-0,212 \%$. Ein Zusammenhang zwischen der Höhe des Ausgangsblutdruckes und der Grösse der Ätherkonzentration, die nötig war, um diesen Blutdruck bis auf die Hälfte zu reduzieren, ist hier nicht nachweisbar. Es geht aus diesen Versuchen hervor, dass das vasomotorische Zentrum der verschiedenen Tiere für Äther sehr verschieden empfindlich ist.

In Versuch III wurde Blut entnommen, nachdem der Blutdruck von 206 auf 155, also auf drei Viertel des ursprünglichen Wertes heruntergegangen war. Der Äthergehalt des Blutes betrug $0,136 \%$. Einen ähnlichen Wert $(0,138 \%)$ gab Versuch XI. Der Blutdruck war anfangs 170 und sank bis auf 126 .

Interessant war Versuch VII, wo bei einem Äthergehalt des Blutes von $0,11 \%$ der Blutdruck noch absolut unverändert geblieben war. In Versuch XII brachte eine sehr hohe Ätherkonzentration $(0,206 \%)$ den Druck nur von 98 auf 74 .

In Versuch I wurde dem Tiere auf einmal eine sehr grosse Menge Äther zugeführt. Der Druck blieb erst konstant, sank dann plötzlich steil ab. Das Blut enthielt in diesem Augenblick 0,18\% Äther.

In sieben Versuchen wurde Blut entnommen, wenn das Herz nahezu stillstand. Die gefundenen (-)-Werte betrugen im Mittel $0,209 \%$, die zwei $(+)$-Werte im Mittel $0,296 \%$. Aus diesen beiden Zahlen lässt sich schliessen, dass ein Äthergehalt von ca. $\mathbf{0 , 2 5 0 \%}$ genügt, um bei den meisten Katzen mit intakten Vagi das Herz zum Stillstand zu bringen. Sehr schön in Übereinstimmung hiermit ist die in Versuch XIII gefundene Zahl. Hier geschah die Blutentnahme sehr schnell unmittelbar vor dem Tode des Tieres, so dass dieser Wert als sehr genau betrachtet werden muss. Fs wurden $\mathbf{0 , 2 5 0} \%$ Äther im Blute gefunden.

In dieser Versuchsreihe konnte beiläufig .der Einfluss der Äthernarkose auf die durch faradisehe Reizung des N. ischiadicus hervorgerufenen Blutdruckänderungen studiert werden. Faradische Reizung dieses Nerven gibt bekanntlich bei den meisten intakten Katzen eine Blutdrucksteigerung. Nach Einschalten der Äthernarkose nimmt die Grösse dieser reflektorischen Blutdrucksteigerung $a b$, und die Steigerung kann in eine reflektorische Senkung des Blutdruckes 
übergehen. In heiden Fällen aber wird schliesslich ein Stadium erreicht, wo faradische Reizung gar keinen Einfluss mehr auf den Blutdruck ausübt. Wurde in diesem Stadium Blut entnommen, so zeigte sich, dass bei Benutzung mittelstarker Ströme $0,11 \%$ und $0,138 \%$ Äther im Blute den Effekt der Reizung aufhoben. Bei Benützung stärkster faradischer Reizung (im primären Kreis ein Akkumulator und maximaler Stand des Induktoriums) betrugen diese Zahlen $0,183,0,206$ und $0,212 \%$. Letzteres heisst also, dass bei einem Äthergehalt des Blutes, welcher unter gewöhnlichen Umständen ein normales Tier töten würde (Werte über $0,17 \%$ ), das vasomotorische Zentrum noch durch Rejzung des $\mathrm{N}$. ischiadicus, wenn auch nur schwach, doch erregt werden kann.

Es hat sich bei dieser Versuchsreihen als oherausgestellt, dass in intakten Tieren mit intakten Vagi ein Äthergehalt des Blutes von $0,250 \%$ genügt, um das Herz zum Stillstand zu bringen. Es ergab sich nun die Frage, ob beim Zustandekommen dieses Herzstillstandes auch eine Wirkung des Äthers auf das Vaguszentrum eine Rolle spielen könnte. Um dieses entscheiden zu können, wurden einige Versuche an Katzen mit durchschnittenen Vagi angestellt.

\section{Versuehe an intakten Katzen mit durchschnittenen Vagi.}

Die Versuchsanordnung war genau dieselbe wie in den vorigen Versuchen, nur wurden bei den Tieren die Vagi durchschnitten. Die Blutentnahme im Augenblicke, wo das Herz stillstand oder nahezu stillstand, geschah ebenfalls genau in derselben Weise, wie oben beschrieben. Dementsprechend sind auch die in diesen Versuchen enthaltenen Ätherwerte mit dem Zeichen ( + ) oder (-) versehen.

Das Resultat der Versuche ist aus Tabelle 3 (S. 138) ersichtlich.

Aus dieser Tabelle ergibt sich, dass der Minimumwert, bei dem das Herz einer Katze (mit durchschnittenen Vagi) zum Stillstand gebracht wurde, im Mittel 0,274\% $(0,268$ und 0,280\%) und der Maximumwert im Mittel $\mathbf{0 , 3 5 3} \%$ beträgt. Es wird also der Äthergehalt, der in den meisten Fällen imstande ist, das Herz zum Stillstand zu bringen, ungefähr bei $\mathbf{0 , 3 1 0} \%$ liegen.

Versuch IX ist einigermaassen als eine Ausnahme zu betrachten, das Versuchstier hatte, wie sich bei der späteren Sektion herausstellte, eine Pneumonie, der Ätbergehalt des Blutes im Moment des Todes war ziemlich niedrig und betrug nur $0,267 \%$. 
138 P. Schram, W. Storm van Leeuwen und M. van der Made:

Tabelle 3.

Versuche an intakten Katzen mit durchschnittenen Vagi. Äthergehalt des Blutes.

\begin{tabular}{|c|c|c|c|c|}
\hline $\begin{array}{l}\text { Versuch } \\
\text { Nr. }\end{array}$ & $\begin{array}{c}\text { Blutdruck bis auf } \\
\left.\text { die Hälfte }{ }^{1}\right) \\
0 / 0\end{array}$ & $\begin{array}{c}\text { Max. faradische } \\
\text { Reizung ohne } \\
\text { Einfluss auf } \\
\text { Blutdruck } \\
\% \%\end{array}$ & $\begin{array}{c}\text { Herz- } \\
\text { stillstand } \\
\%\end{array}$ & $\begin{array}{c}\text { An- } \\
\text { merkungen }\end{array}$ \\
\hline IV & $\frac{198}{93} \quad 0,175$ & - & $0,268(-)$ & \\
\hline $\mathrm{V}$ & $\frac{200}{107} \quad 0,233$ & - & $0,351(+)$ & \\
\hline VI & $\frac{128}{60} \quad 0,109$ & - & $0,28 \quad(-)$ & \\
\hline IX & - & - & $(0,267)$ & Pneumonie \\
\hline $\mathrm{X}$ & $\frac{200}{90} \quad 0,186$ & 0,186 & $0,354(+)$ & \\
\hline
\end{tabular}

Wie aus Spalte 2 ersichtlich ist, haben wir in vier Fällen den Äthergehalt des Blutes im Augenblicke bestimmt, wo die Narkose derart schädigend auf den Kreislauf eingewirkt hatte, dass der Blutdruck bis auf zirka die Hälfte des ursprünglichen Wertes gesunken war. Weil in diesem Falle arterielles Blut entnommen wurde, während das Herz noch sehr kräftig schlug, so sind die hierbei gewonnenen Zahlen viel genauer als die obigen. Trotzdem sind die Differenzen zwischen den bei diesen vier Fällen gefundenen Werten sehr gross. In Versuch VI war der Anfangsblutdruck 128, ein Äthergehalt von $0,109 \%$ genügte schon, um diesen Druck auf 60 herunterzubringen. Offenbar war das vasomotorische Zentrum dieses Tieres sehr leicht beeinflussbar. Dass nicht Schwäche des Herzens die Ursache war, zeigt der Umstand, das die tödliche Konzentration für das Herz eine relativ hohe war $((-)$-Wert $0,280 \%)$.

In Versuch $\mathrm{V}$ war zur Herabsetzung des Blutdruckes von 200 auf 107 eine Ätherkonzentration von $0,233 \%$ nötig, also mehr als die doppelte Menge wie diejenige aus Versuch IX.

Die Zahl dieser Versuche ist natürlich zu gering, um aus ihnen weitgehende Schlüsse ziehen zu können; nur sei bemerkt, dass in den Fällen mit hohen Ätherwerten der Anfangsblutdruck hoch war und in den Fällen mit niedrigen Ätherwerten auch der Anfangs-

1) Die Bruchzahlen beziehen sich auf den Blutdruck. Der Zähler gibt den Anfangsblutdruck, der Nenner den Blutdruck im Augenblick der Blutentnahme 
blutdruck niedrig war. Wie dem auch sei, auf jeden Fall geht aus diesen Versuchen hervor, dass das vasomotorische Zentrum der verschiedenen Tiere für Äther sehr verschieden empfindlich ist.

Wie zu erwarten war, sind die Ätherkonzentrationen, welche beim intakten Tiere mit durchschnittenen Vagi das Herz zum Stillstand bringen, niedriger als die im vorigen Abschnitt für die isolierte Herz-Lungenzirkulation gefundenen. Indessen nähern sich die höchsten Zahlen am intakten Tiere $(0,353 \%)$ den niedrigsten Zahlen, welche am Starling-Apparat gewonnen wurden $(0,330 \%)$.

Nachdem in den oben beschriebenen Versuchen sich herausgestellt hatte, dass das Herz von Katzen mit durchschnittenen Vagi resistenter gegen Äther ist als das Herz von Katzen mit intakten Vagi, war noch festzustellen, ob die gleiche Erhöhung der Resistenz gegen Äther sich erreichen liesse durch Injektion mit Atropin bei einem Tier mit intakten Vagi. Wir haben fünf Versuche in dieser Richtung angestellt. Die Versuchsanordnung war wieder genau dieselbe wie in den beiden vorigen Serien, nur wurde den Katzen vor dem Anfang des Versuches 2-21/2 mg Atropin intravenös injiziert.

Das Resultat dieser. Versuche ist aus Tabelle 4 ersichtlich.

Tabelle 4 .

Versuche an intakten Katzen mit intakten Vagi nach Einspritzung von Atropin. Äthergehalt des Blutes.

\begin{tabular}{c|c|c|c}
\hline $\begin{array}{c}\text { Versuch } \\
\text { Nr. }\end{array}$ & $\begin{array}{c}\text { Blutdruck bis auf } \\
\text { zirka ein Drittel } \\
\text { \% }\end{array}$ & $\begin{array}{c}\text { Herzstillstand } \\
\%\end{array}$ & Anmerkungen \\
\hline XIV & - & $0,247(-)$ & \\
XV & - & $0,333( \pm)$ & \\
XVI & $\frac{106}{36} 0,28$ & $0,291( \pm)$ & \\
XVII & 0,275 & $0,356(+)$ & Ausgebreitete Pneumonie. \\
XVIII & - & 0,126 & A
\end{tabular}

In Versuch XV und XVI geschah die Blutentnahme sehr schnell, in einem Stadium, wo das Herz gerade noch schlug, und es müssen diese Werte deshalb als sehr exakt betrachtet werden; der Äthergehalt des Blutes betrug $0,333 \%$ und $0,291 \%$, im Mittel also $0,312 \%$. Der Wert aus Versuch XVII war zu hoch $(0,356 \%)$, in diesem Versuch schlug das Herz bei $0,275 \%$ sicher noch. Versuch XIV gibt eine zu niedrige Zahl. Das Mittel von Versuch XVII und XIV 
beträgt aber wieder ca. 0,300\%. Aus diesen vier Versuchen kann also geschlossen werden, dass bei Katzen mit intakten Vagi nach Einspritzung von Atropin ein Äthergehalt von ca. $0,310 \%$ im Blute erforderlich ist, um das Herz zum Stillstand zu bringen. Es stimmt dieser Wert vollkommen überein mit den an Katzen mit durchschnittenen Vagi gewonnenen Zahlen. Man muss aus diesen letzten Versuchen also wohl schliessen, dass während tiefer Narkose dem Herzen schädliche Einflüsse durch die Nn. vagi zufliessen. Dass in tiefer Narkose der $\mathrm{N}$. vagus noch erregbar ist, geht auch aus den Versuchen von Elfstrand ${ }^{1}$ hervor. Elfstrand hat das Verhalten der Vaguserregbarkeit während der Narkose an Kaninchen studiert und fand, dass während tiefer Narkose die Erregbarkeit des Vagus zwar abnimmt, so dass durch faradische Reizung dieses Nerven kein Herzstillstand mehr bervorgerufen werden kann, dass aber in tiefster Narkose noch ein deutlicher Einfluss durch Vagusreizung auf den Blutdruck ausgeubt werden kann.

Der Unterschied, der in unseren Versuchen in der $\cdot$ Resistenz gegen Äther zwischen Tieren mit und ohne Vagotomie gefunden wurde, stimmt mit den Erfahrungen von $\mathrm{Knoll}^{2}$ ) überein. Knoll studierte bei Kaninchen die Wirkung von Äther und Chloroform auf Kreislauf und Atmung. Das Narkotikum wurde den Tieren mit der Einatmungsluft zugeführt. Um die Nachteile der auf die Vagotomie folgenden Verengerung der Stimmritze auszuschalten, wurde bei den Tieren eine Trachealfistel angelegt. Gelegentlich wurde auch künstliche Atmung angewandt. Knoll fand nun, dass Chloroform von Tieren mit durchsehnittenen Vagi schlechter ertragen wird als von Tieren mit intakten Vagi. Äther dagegen wurde von den vagotomierten Tieren besser vertragen. Letzteres steht also mit unseren Erfahrungen in Einklang.

Anhangsweise sei noch das Ergebnis von Versuch XVIII erwähnt. Nach Einschalten einer ziemlich hohen Ätherkonzentration in der Einatmungsluft sank hier der Blutdruck ausserordentlich rasch und steil herunter, so dass innerhalb $2^{1 / 2}$ Minuten Herzstillstand ein-

1) M. Elfstrand, Beobachtungen über die Wirkungen einiger aliphatischer Kohlenwasserstoffe, verglichen mit derjenigen des Äthers, und über das Verhalten der Vaguserregbarkeit während der Narkose. Arch. f. exper. Pathol. u. Pharm. Bd. 43 S. 435. 1900.

2) P. Knoll, Über die Wirkung von Chloroform und Äther auf Atmung und Blutkreislauf. Ber. d. Wiener Akad. 3. Abt. Bd. 78 S. 223.1878. 
getreten war. Das in diesem Augenblick entnommene Blut enthielt nur 0,126\% Äther. Bei der Sektion stellte sich heraus, dass dieses Tier an einer ausgebreiteten Pneumonie gelitten hatte. Der Verlauf der Blutdrucksenkung dieses Versuches ist aus Fig. 3 ersichtlich. Zum Vergleich ist in Fig. 4 der Verlauf des Blutdruckes aus Versuch XVII gegeben. In beiden Fällen handelte es sich um Tiere mit intakten Vagi, welche vor dem Versuch $2 \frac{1 / 2}{\mathrm{mg}}$ Atropin intravenös eingespritzt bekommen hatten. Die mit der künstlichen Atmung zugeführte Ätherkonzentration war auch in beiden Fällen die gleiche.

Wiewohl also beim Narkotisieren eines intakten Tieres weit niedrigere Äthermengen schon genugen, um das Herz $\mathrm{zu}$ sçhädigen resp. zum Stillstand zu bringen, als bei Versuchen am St arling-Apparat, so geht aus den Versuchen dieses Abschnittes doch hervor, dass die Ätherkonzentrationen, welche bei unserer Versuchsanordnung das Herz zum Stillstand bringen, immer noch beträchtlich höher liegen (Minimum 0,250\%) als die durch Nicloux und uns im Moment des Todes gefundenen $(0,170 \%)$. Weiter lässt sich aus denVersuchen an intakten'Tieren mit intaktenVagi schliessen, dass

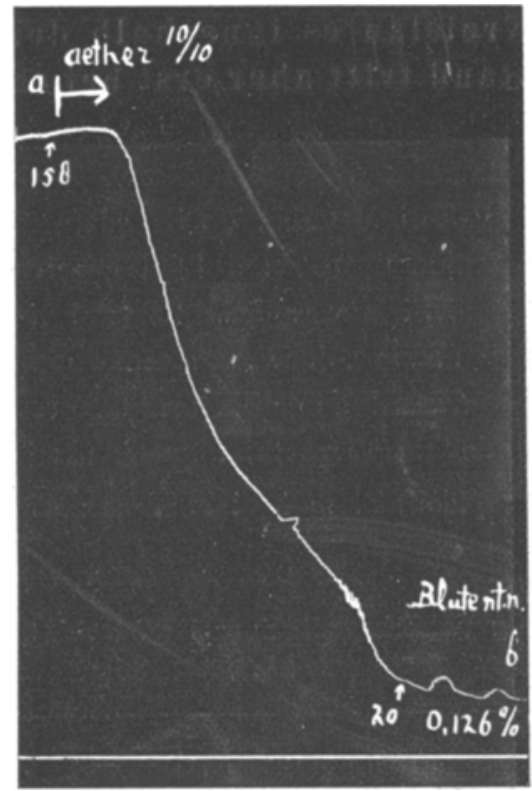

Fig. 3. Intakte Katze. 21/2 mg Atropin. Versuch XVIII. Registrierung des Blutdruckes. Bei $a$ 10/10 Äther mit der künstlichen Atmung zugeführt. Der Blutdruck sinkt steil von $158 \mathrm{~mm} \mathrm{Hg}$ auf $20 \mathrm{~mm}$. Sektion: Ausgebreitete Pneumonie. (Auf $4 / 5$ verkleinert.)

bei Ätherkonzentrationen im Blute, welche niedriger als die von $\mathrm{Ni}$ cl o u $\mathrm{x}$ gefundenen sind, und also von einer Grössenordnung sind, welche im Toleranzstadium der Narkose wiederholt erreicht werden muss, schon einen deutlichen Einfluss auf den Kreislauf ausgeübt werden kann. Namentlich kann der Blutdruck bei diesen Konzentrationen beträchtlich herabgesetzt sein (Versuch II, III, VII und XI!). Diese Blutdrucksenkung beruht offenbar auf Schädigung des vasomotorischen Zentrums, da das Herz erst bei höhereu Konzentrationen geschädigt 
142 P. Schram, W. Storm van Leeuwen und M. vagder Made:

wird. Das Ergebnis der Atropinversuche eröffnet die Möglichkeit, den schädigenden Einfluss auf das Herz zu vermindern.

Bei intakten Katzen mit intakten Vagi und bei künstlicher Atmung ist also eine Ätherkonzentration im Blute von $0,101-0,212 \%$ imstande, den Kreislauf zu schädigen. Herzstillstand wird bei $\mathbf{0 , 2 5 0} \%$ hervorgerufen. Bei intakten Tieren mit ausgeschalteten Vagi schwanken die Zahlen für die Schädigung des Kreislaufes innerhalb derselben Grenze. Herzstill. stand tritt aber erst bei ca. $\mathbf{0 , 3 1 0} \%$ auf.

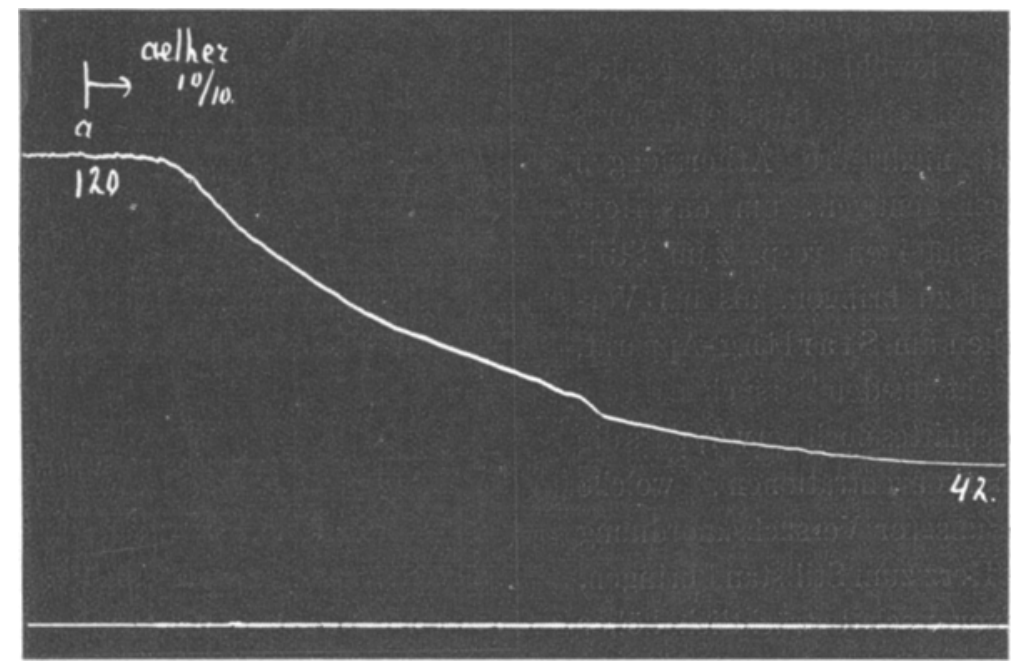

Fig. 4. Intakte Katze. 21/2 mg Atropin.

Versnch XVII. Registrierung des Blutdruckes. Bei $a{ }^{10} / 10$ Äther mit der künstlichen Atmung dem Tiere zugeführt. Der Blutdruck sinkt viel allmählicher als in Fig. 3. Sektion: Vollkommen gesundes Tier. (Auf $4 / 5$ verkleinert.)

\section{Versuche am isolierten Katzenherzen.}

$$
\text { Von }
$$

\section{W. Storm van Leeuwen und M. van der Made.}

Aus den bisher beschriebenen Untersuchungen ist hervorgegangen, dass die letale Ätherdosis für das Herz in Versuchen am isolierten Herz-Lungenkreislauf nach Starling viel höher ist als bei Versuchen am intakten Tiere. Der Grund dieses Unterschiedes ist wie schon oben betont wurde - der Umstand, dass bei Versuchen am Starling-Apparat der Blutdruck bis zuletzt konstant bleibt, 
während bei Versuchen am intakten Tiere der Blutdruck nach der Ätherzufuhr : allmählich sinkt und also zu der Ätherwirkung noch der ungünstige Einfluss eines niedrigen Blutdruckes und der hierdurch verursachten ungenügenden Ernährung des Herzens kommt. Es lag pun auf der Hand, zu untersuchen, ob das Katzenherz, wenn es unter noch günstigere Ernährungsbedingungen gebracht würde als bei Versuchen am Starling-Apparat, noch höhere Ätherkonzentrationen ertragen würde. $\mathrm{Zu}$ diesem $\mathrm{Zwecke}$ haben wir einige Versuche an isolierten Katzenherzen angestellt, welche wach dem Langend orffschen Verfahren mit unverdünntem Blute durchströmt wurden. Diesem Blute wurden steigende Mengen Äthers zugeführt, und es wurden die Grenzkonzentrationen bestimmt, welche das Herz zu schädigen und welche es zum Stillstand zu bringen imstande waren. Eine derartige Untersuchung war um so mehr erwünscht, als Dieballa ${ }^{1}$ ) und $\mathrm{Loeb}^{2}$ ) in ihren Versuchen an isolierten Frosch- resp. Warmblüterherzen Äthermengen gefunden haben, welche die in den vorigen Abschnitten in Betracht kommenden Zahlen weit überschritten. Di eballa hatte die Herzen mit NaClLösung durchströmt, L o e b mit Blut-Kochsalzgemisch gearbeitet, und es erhob sich die Frage, ob dieser Umstand die grossen Untersehiede zwischen den von uns und von den beiden erwähnten Autoren gefundenen Zahlen restlos erklären könnte.

Wir können über neun gelungene Versuche am LangendorffApparat berichten.

Es wurde immer mit Katzenherzen gearbeitet, die mit unverdünntem, defibriniertem Blute durchströmt wurden. Die Temperatur des Durchströmungsblutes wurde in jedem einzelnen Versuch bis auf $0,5^{\circ}$ konstant gehalten und schwankte in den verschiedenen Versuchen zwischen $85^{\circ}$. und $37^{\circ}$. Um das unverdünnte Blut mit genügender Geschwindigkeit durch das Koronarsystem pressen zu können, musste dasselbe unter ziemlich hohen Druck gebracht werden, manchmal bis zu $200 \mathrm{~mm} \mathrm{Hg}$. Während der Dauer eines Versuches blieb der Druck konstant.

Es wurden in diesen Versuchen die Ausschläge eines Vorhofs und der Kammern getrennt registriert. Nachdem bei Durchströmung mit ätherfreiem Blute eine genügend lange Normalperiode geschrieben worden war, wurde auf ätherhaltiges Blut umgeschaltet und der Effekt beobachtet. Herzstillstand wurde angenommen, wenn innerhalb einiger Minuten, nachdem das ätherhaltige Blut das Herz erreicht batte, das. Herz zu schlagen aufhörte.

1) Dieballa, l. c.

2) 0. Loeb, I. c. 
144 P. Schram, W. Storm van Leenwen und M. vander Made:

Nach Beendigung eines Versuches wurde der Äthergehalt des Durchströmungsblutes bestimmt. Hierzu wurde immer eine Blutprobe aus dem Reservoir genommen, also immer Blut benutzt, welches das Herz noch nicht erreicht hatte. Deshalb wurden immer genaue Werte erbalten.

Das Resultat dieser Versuchsreihe ist aus Tabelle 5 ersichtlich.

Tabelle 5 .

Versuche. an isolierten Katzenherzen bei Durchströmung mit unverdunntem Blute. Äthergehalt des Blutes.

\begin{tabular}{|c|c|c|c|c|c|}
\hline $\begin{array}{c}\text { Versuch } \\
\text { Nr. }\end{array}$ & $\begin{array}{c}\text { Kein } \\
\text { Einfluss auf } \\
\text { das Herz } \\
\%\end{array}$ & $\begin{array}{c}\text { Sehr } \\
\text { geringer } \\
\text { Einfluss } \\
\%\end{array}$ & $\begin{array}{c}\text { Starker Einfluss. } \\
\text { Kontraktionen } \\
\text { bis auf die } \\
\text { Hälfte bis ein } \\
\text { Viertel } \\
\% \%\end{array}$ & $\begin{array}{c}\text { Stärkste } \\
\text { Schädigung. } \\
\text { Nahezu Herz- } \\
\text { stillstand } \\
\%\end{array}$ & $\begin{array}{c}\text { Herz- } \\
\text { stillstand } \\
\%\end{array}$ \\
\hline $\begin{array}{r}\text { I } \\
\text { II } \\
\text { III } \\
\text { IV } \\
\text { V } \\
\text { VI } \\
\text { VII } \\
\text { VIII }\end{array}$ & $\begin{array}{l}0,077 \\
= \\
- \\
- \\
-\end{array}$ & $\begin{array}{l}0,286 \\
- \\
- \\
\overline{-} \\
0,28 \\
-\end{array}$ & $\begin{array}{l}\overline{ } \\
\overline{-} \\
\overline{-} \\
\overline{0}, 384 \\
0,389\end{array}$ & $\begin{array}{l}\bar{z} \\
\bar{z} \\
0,506 \\
\overline{-} \\
=\end{array}$ & $\begin{array}{c}\overline{0} \\
0,525 \\
0,560 \\
0,576 \\
- \\
- \\
-\end{array}$ \\
\hline
\end{tabular}

$\mathrm{Zu}$ dieser Tabelle ist folgendes zu bemerken.

Ein Äthergehalt von $0,28 \%$ hat bei Durchströmung des isolierten Katzenherzens nur einen sehr geringen Einfluss. Eine deutlich schädliche Wirkung wird hervorgerufen durch Konzentrationen, die etwas unterhalb $0,40 \%$ liegen. Das sind also Konzentrationen, welche bei Versuchen am Starling-Apparat das Herz fast zum Stillstand brachten. Ein Beispiel vom Einfluss einer Ätherkonzentration von $0,384 \%$ gibt Fig. 5. Herzstillstand wurde in drei Versuchen erreicht mit Ätherwerten, welche im Mittel 0,553\% betrugen. Ein Beispiel des Herzstillstandes durch Äther gibt Fig. 6. Der Ventrikel stand in diesem Versuch fast sofort nach dem Einströmen des ätherhaltigen Blutes still, das Atrium schlug noch etwas länger weiter. Wir verfugen aber auch abber Versuche, wo bei einer ähnlichen Konzentration Ventrikel und Atrium gleichzeitig stillstanden.

Bei Versuchen am Langendorf-Apparat hat also ein Äthergehalt von $0,280 \%$ nur eine sehr geringe, $\mathbf{0 , 3 9 0} \%$ eine deutlich schädigende Wirkung auf das Herz. Eine Konzentration von ca. 0,55\% bringt das Herz schnell zum Stillstand. 
Fasst man das Resultat der drei Abschnitte zusammen, so ergibt sich folgendes : a) Diejenigen Ätherkonzentrationen, welche im Toleranz-
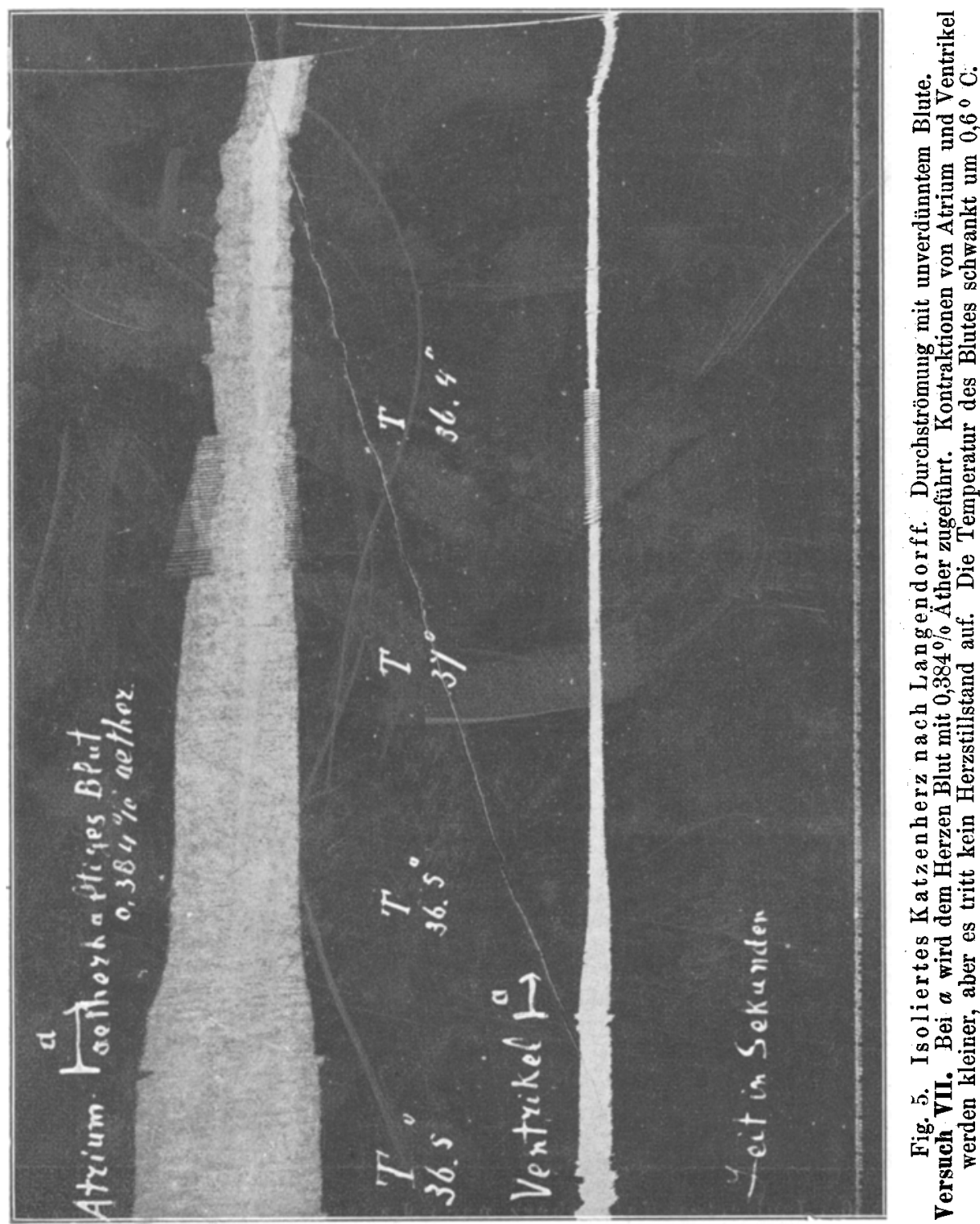

stadium der Äthernarkose bei der Katze im Blute gefunden werden, schädigen den Kreislauf wenig und das Herz beinahe gar nicht. b) Diejenigen Ätherkonzentrationen, welche imstande sind, ein norPflüger's Archì für Physiologie. Bd. 165. 
146 P. Schram, W. Storm van Leeuwen und M. van der Made:

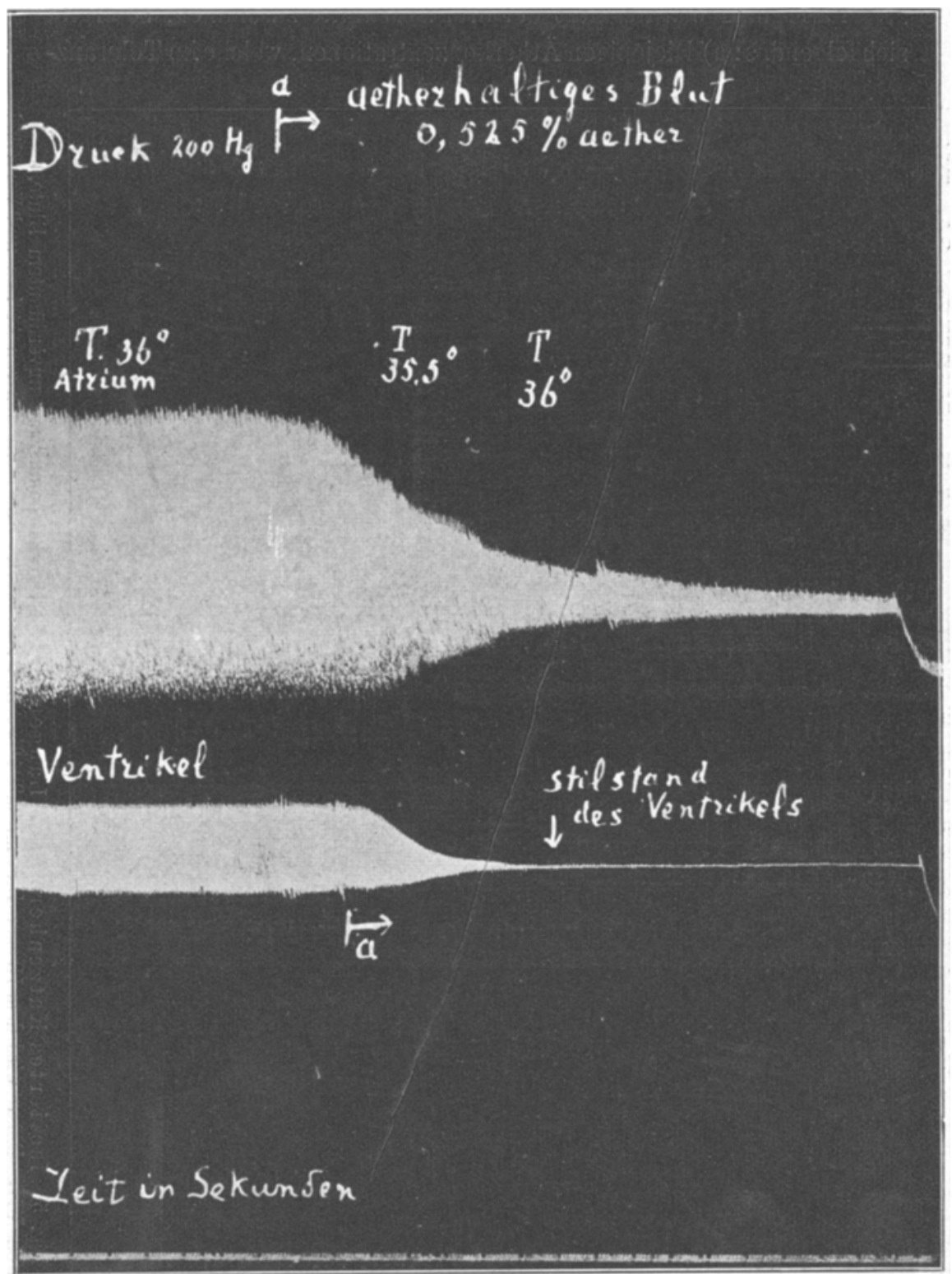

Fig. 6. Isoliertes Katzenherz nach Langendorff. Durcbströmung mit unverdünntem Blute.

Versuch II. Bei $a$ wird dem Herzen Blut mit $0,525 \%$ Äther zugeführt. Der Ventrikel steht fast sofort still. Das Atrium macht noch kleine Kontraktionen, welche aber auch nach einigen Minuten aufhören. Die Temperatur des Blutes schwankt um $0,5^{\circ} \mathrm{C}$. 
males Tier zu töten, üben auf den Blutdruck einer intakten künstlich geatmeten Katze einen deutlich schädigenden Einfluss aus, und zwar sowohl durch Schädigung des vasomotorischen Zentrums als durch Vaguswirkung auf das Herz. Das Herz selbst wird bei derartigen Konzentrationen direkt nur schwach beeinflusst. c) Die Ätherkonzentration; welche das Herz eines intakten Tieres bei künstlicher Lungenventilation tötet, beträgt $0,250 \%$. Es wird aber ein solches Herz nicht durch den Äther allein zum Stillstand gebracht, sondern auch andere Faktoren wirken mit; an erster Stelle Einflusse, welche durch die Nn. vagi dem Herzen zufliessen. Werden diese Einflisse durch Injektion von Atropin oder Durchschneidung der Vagi aufgehoben, so erträgt das Herz Dosen bis zu 0,310\%. Aber auch diese Konzentration würde das Herz nicht töten, wenn sich zu dem schädlichen Einfluss des Äthers nicht noch die infolge des niedrigen Blutdruckes auftretende mangelhafte Ernährung des Herzens gesellen würde. Wird letzterer Einfluss ausgeschaltet wie in Versuchen am Starling-Apparat oder am nach Langend orff durchströmten Herzen, so lässt sich die minimal letale Dosis für das Herz auf $0.40 \%$ bzw. $0,55 \%$ steigern.

Schliesslich sei bemerkt, dass die von uns am isolierten Herzen gewonnenen Ätherzahlen, verglichen mit den von Sherrington und S owton ${ }^{1}$ ) unter denselben Umständen gefundenen Chloroformwerten, das Verhältnis des Wirkungsgrades von Äther und Chloroform ergeben. Sherrington und Sow to $n$ fanden als minimal letale Dosis in unverdünntem Blute für Chloroform $0,1 \%$. Wir fanden für Äther $0,55 \%$. Hieraus ergibt sich durch eine einfache Berechnung, dass, um dieselbe Wirkung auf das Warmblüterherz hervorzurufen, von Äther neunmal stärkere Molekularkonzentrationen erforderlich sind als von Chloroform. Wird aber hierbei in Betracht gezogen, dass Äther sich in ganz anderer Weise zwischen Blutkörperchen und Plasma verteilt als Chloroform, so ändert sich dieses Verhältnis sehr. Wie schon aus Versuchen von $\mathrm{Poh}{ }^{2}$ ) hervorgeht, verbindet sich in einer Lösung von Chloroform in Blut das Chloroform "vorwiegend mit den morphotischen Bestandteilen, also mit den

1) C. S. Sherrington and S. C. M. Sowton, 1. c.

2) J. Pohl, Über Aufnabme und Verteilung des Chloroforms im tierischen Organismus. Arch. f. exper. Pathol. u. Pharmak. Bd. 28 S. 239.1891. 
roten Blutzellen". Nicloux ${ }^{1}$ ) hat später nachgewiesen, dass in einer Lösung von Chloroform in Blut $88 \%$ des Chloroforms sich in den Blutzellen befinden und nur $12 \%$ im Plasma zu finden sind. Für Äther liegen die Verhältnisse anders. Äther ist in Wasser viel besser löslich als Chloroform, und es ist - nach Nicloux - im Blute nur zu $52 \%$ an die Blutzellen und zu $48 \%$ im Plasma gebunden. Zwar gibt $\mathrm{Frant} \mathrm{z}^{3}$ ) an, dass die Lösungsverhältnisse des Chloroforms sich auf Äther ausdehnen lassen, und dass es "den Eindruck macht, dass die Bindung desselben (des Äthers) eine innigere ist als die des Chloroforms". Aber die Ätherbestimmungen von Frantz sind viel weniger genau als die von Nicloux. Man darf also wohl annehmen, dass das Äther sich im Blute ungefähr gleichmässig auf Blutzellen und Plasma verteilt. Weil also von im Blute vorhandenem Chloroform das Plasma vur $12 \%$ enthält, während von Äther $48 \%$ im Plasma sich befinden, so ist das wirkliche Verhältnis der Wirkungsgrade zwischen Äther und Chloroform nicht wie $1: 9$, sondern wie $\left.\frac{12 \times 1}{100}: \frac{48 \times 9}{100}=1: 36^{4}\right)$, das heisst also, dass in dieser Beziehung ein Chloroformmolekül 36 Äthermolekülen gleichwertig ist. Dieballa hatte auf Grund seiner Versuche am mit NaClLösung durchspülten Froschherzen ein Verhältnis von $1: 36$ gefunden. Loeb fand nach Versuchen an isolierten, mit Blut-Kochsalzgemisch durchströmten Säugetierherzen ein Verhältnis von $1: 35$.

Das von uns gefundene Wirkungsverhältnis zwischen Äther und Chloroform stimmt also mit dem von Dieballa nnd Loeb überein.

L o eb hat bei seiner Berechnung den Unterschied in der Verteilung (auf Blutkörperchen und Plasma) zwischen Äther und Chloroform nicht berücksichtigt. Überdies sind seine absoluten Zahlen viel höher als die unserigen, so dass ein Vergleich zwischen seinen Versuchen und denen der vorliegenden Arbeit nicht möglich ist.

1) M. Nicloux, l. c. \$. 37.

2) M. Nicloux, l. c. S. 91 .

3) R. Frantz, Über das Verhalten des Äthers im tierischen Organismus. Inaug.-Diss. Würzburg 1895.

4) In der vorhergehenden Arbeit wurde für die Aufhebung des homolateralen Beugereflexes der Katze ein Wirkungsverhältnis zwischen Chloroform und Äther von $1: 50$ gefunden. 
Schlusssätze.

1. Beim Narkotisieren einer intakten Katze mit Äther unter künstlicher Atmung tritt Herzstillstand auf bei einem Äthergehalt des Blutes von $\mathbf{0 , 2 5 0} \%$; werden Einflüsse des Vaguszentrums ausgeschlossen durch Atropin oder Vagotomie, so beträgt die minimal letale Dosis für das Herz $\mathbf{0 , 3 1 0} \%$.

2. Wird der schädliche Einfluss der in tiefer Narkose erfolgenden Blutdrucksenkung ausgeschaltet und das Herz am St a rling-Apparat unter günstigere Ernährungsbedingungen gebracht, so steigt die minimal letale Konzentration auf ca. $\mathbf{0 , 4 4} \%$.

3. Wird die Ernährung des Herzens noch besser gesichert und das isolierte Katzenherz nach $L$ angendorff mit unverdünntem Blute durchströmt, so wird die minimal letale Konzentration $\mathbf{0 , 5 5 3} \%$.

4. Das Verhältnis der Wirkungsgrade von Äther und Chloroform beträgt, ausgedrückt in Molekularkonzentrationen, unter Berücksichtigung des verschiedenartigen Verteilungsmodus dieser Narkotika auf Blutkörperchen und Plasma nach Versuchen mit unverdünntem Blute $1: 36$.

5. Bei Ätherkonzentrationen im Blute, wie sie beim Narkotisieren normaler Tiere in Betracht kommen $(\mathbf{0 , 1 3 - 0 , 1 4 \% )}$, kann, besonders in Fällen, wo der Anfangsblutdruck hoch war, eine Blutdrucksenkung auftreten, wobei der Blutdruck bis auf drei Viertel des Anfangswertes (in einem Fall bis auf die Hälfte) heruntergehen kann. Diese Senkung wird verursacht durch Beeinflussung des vasomotorischen Zentrums und Vaguswirkung. Der Herzmuskel wird durch diese Ätherkonzentrationen noch kaum geschädigt.

6. Bei Ätherkonzentrationen, welche im Blute totnarkotisierter Tiere gefunden werden (ca. 0,17\%), ist der Kreislauf einer Katze, deren Lungen künstlich ventiliert werden, meistens schon deutlich geschädigt. Auch diese Ätherkonzentrationen wirken auf den Herzmuskel noch wenig ein. 